\title{
In Vitro Evaluation of Humanized/De-immunized Anti-PSMA Immunotoxins for the Treatment of Prostate Cancer
}

\author{
MARTA MICHALSKA, SUSANNE SCHULTZE-SEEMANN, IRINA KUCKUCK and PHILIPP WOLF \\ Department of Urology, Medical Center - University of Freiburg, \\ Faculty of Medicine, University of Freiburg, Freiburg, Germany
}

\begin{abstract}
Background: We generated humanized/deimmunized immunotoxins targeting the prostate-specific membrane antigen (PSMA) and tested their cytotoxic activity against prostate cancer cells in vitro. Materials and Methods. The humanized/de-immunized version of our murine antiPSMA single-chain antibody fragment ( $s c F v)$ D7, termed $h D 7-1(V L-V H)$, was ligated to the 40-kDa toxin domain of Pseudomonas aeruginosa exotoxin A (PE40), and to the deimmunized 24-kDa toxin domains PE24 or PE24mut. The immunotoxins designated as hD7-1(VL-VH)-PE40, hD7-1(VL$V H)-P E 24$ and hD7-1(VL-VH)-PE24mut were bacterially expressed and purified by affinity chromatography. Binding and cytotoxicity were examined by flow cytometry and viability assay, respectively. Results: All immunotoxins revealed strong binding to prostate cancer cells expressing PSMA and specific cytotoxicity, with half-maximal inhibitory concentration values in the picomolar range. Conclusion: We successfully created powerful anti-PSMA immunotoxins with reduced immunogenicity for further clinical development and application against advanced prostate cancer.
\end{abstract}

Current treatment against advanced prostate cancer still requires improved progressive concepts with enhanced efficacy. One possibility is the development of immunotoxins against the prostate-specific membrane antigen (PSMA), which has been demonstrated to be an excellent target in various preclinical and clinical trials against prostate cancer (1-5).

By our group, immunotoxins were constructed by recombinant fusion of the anti-PSMA single chain antibody

This article is freely accessible online.

Correspondence to: Philipp Wolf, Department of Urology, Medical Center - University of Freiburg, Faculty of Medicine, University of Freiburg, Breisacher Str. 66, 79106 Freiburg, Germany. Tel: +49 76127028921, e-mail: philipp.wolf@uniklinik-freiburg.de

Key Words: Immunotoxin, Pseudomonas exotoxin A, deimmunization, PSMA, prostate cancer. fragment $(\mathrm{scFv})$ D7 to the truncated toxin domain of Pseudomonas aeruginosa exotoxin A (PE), called PE40 $(6,7)$. The scFv D7 was derived from our murine monoclonal antibody $(\mathrm{mAb})$ to PSMA 3/F11 $(8,9)$. The PE40 domain consists of the structural domains II, Ib and III (Figure 1). Domain II, designated as the transmembrane domain, is responsible for permeabilization through cellular membranes and cytosolic localization. Domains Ib and III cause ADPribosylation of the elongation factor 2 (eEF2) on the ribosomes, resulting in inhibition of protein biosynthesis and effective cell death (10).

Despite their powerful cytotoxic features, a major problem of PE-based immunotoxins is their high immunogenicity in patients, which is the result of the non-human origin of the antibody-binding domain and the bacterial background of the toxin domain. This makes such immunotoxins inappropriate for clinical application (11). Since multiple immunotherapy cycles are a prerequisite in achieving clinical responses, repeated applications of immunogenic immunotoxins might lead to an activation of the patient's immune system with consequent formation of anti-drug antibodies (ADA), thereby triggering severe side-effects $(12,13)$. Therefore, many attempts have been made to diminish the immunogenicity of immunotoxins by humanization/de-immunization of the antibody binding domains $(14,15)$, modification with macromolecules (16-19), or structural changes of the PE domain (20-22). Several preclinical studies described that deletions or mutations within the PE toxin domain led to the removal of immunodominant Band T-cell epitopes and successfully contributed to diminished immunogenicity and reduced ADA production (20-22).

The goal of the present work was to generate less immunogenic anti-PSMA immunotoxins by use of a humanized/de-immunized anti-PSMA $\mathrm{scFv}$ as binding domain and de-immunized PE variants as toxin domains, and to test their binding and cytotoxic efficacy on prostate cancer cells.

\section{Materials and Methods}

Cell lines. The androgen-sensitive PSMA-expressing prostate cancer cell line LNCaP, its androgen-independent subline C4-2, and the PSMA-negative line DU145 (ATCC, Manassas, VA, USA) were 
cultured in RPMI 1640 medium (Gibco, Invitrogen, Karlsruhe, Germany) with $10 \%$ fetal calf serum (Biochrom, Berlin, Germany) with addition of streptomycin $(100 \mathrm{mg} / \mathrm{l})$ and penicillin $(100 \mathrm{U} / \mathrm{ml})$ at $37^{\circ} \mathrm{C}$ with $5 \% \mathrm{CO}_{2}$. Cell lines were authenticated by short tandem repeats (STR) analysis (Cell Lines Services GmbH, Eppelhein, Germany). Anti-PSMA mAbs 3/F11 and J591 (ATCC, Manassas, VA, USA) were purified from hybridoma supernatant by protein G-sepharose chromatography (GE Healthcare Biosciences AB, Uppsala, Sweden) and dialyzed against phosphate-buffered saline (PBS). Protein content was determined using a Nanodrop Lite Spectrophotometer (Thermo Fisher Scientific, Darmstadt, Germany).

Construction and purification of the humanized/de-immunized antiPSMA scFv and immunotoxins. Humanized/de-immunized versions of the variable domains of the heavy (VH) and light (VL) chains of the murine anti-PSMA scFv D7 were designed by in silico modeling (Lonza Biologics Company, Cambrige, UK). Both chains were synthesized (Gene Art Gene Synthesis, Thermo Fisher Scientific, Regensburg, Germany) and cloned in a VL-VH orientation into the vector pHOG21 containing a flexible glycine-serine $\mathrm{G}_{4} \mathrm{~S}$ linker and $C$-terminal human c-myc and hexahistidine tags for protein detection and purification. The $\mathrm{scFv}$ was called hD7-1(VL-VH).

For the construction of the immunotoxin hD7-1(VL-VH)-PE40, the DNA sequence encoding $P$. aeruginosa exotoxin domain PE40 was fused onto the $C$-terminus of the scFv (Figure 1A). To reduce the immunogenicity of the PE domain, the transmembrane domain II (aa 251-364) was partially deleted, with exception of the furincleavable site (aa 274-284, RHRQPRGWEQL), resulting in a 24 $\mathrm{kDa}$ toxin domain, called PE24. Domain III of the PE24 domain was further de-immunized by the insertion of the following mutations: R427A, R458A, D463A, R467A, R490A, R505A, R538A (20). This variant was named PE24mut. The DNA of the PE24 and PE24mut domains was optimized for Escherichia coli expression, synthesized (Gene Art Gene Synthesis) and $C$-terminally cloned to the scFv hD7-1(VL-VH). This resulted in the immunotoxins hD7-1(VL-VH)-PE24 (Figure 1B) and hD7-1(VL$\mathrm{VH}$ )-PE24mut (Figure 1C). Sequences of all immunotoxins were proved for correctness by Sanger sequencing (GATC, Konstanz, Germany)

The scFv and the immunotoxins were expressed in the periplasm of $E$. coli XL-1 blue and purified by immobilized metal affinity chromatography (IMAC), as described previously (23). Protein content was determined using a Nanodrop Lite Spectrophotometer (Thermo Fisher Scientific).

Sodium dodecyl sulfate polyacrylamide gel electrophoresis (SDS$P A G E$ ). Purity and size of the anti-PSMA scFv and immunotoxins were determined by SDS-PAGE (Invitrogen, Carlsbad, CA, USA) as described earlier (6). The SDS gels were stained using Coomassie Brillant Blue (InstantBlue Protein Stain, Expedeon; Biozol Diagnostica Vertrieb GmbH, Eching, Germany).

Flow cytometry. Binding of the anti-PSMA scFv and immunotoxins was characterized utilizing PSMA-positive $\mathrm{LNCaP}$ and $\mathrm{C} 4-2$ cells, as well as PSMA-negative DU145 cells by flow cytometry. For this, $2 \times 10^{5}$ cells in PBS (with $3 \%$ fetal bovine serum and $0.1 \%$ sodium azide) were incubated with anti-PSMA $\mathrm{scFv}$ or immunotoxins at different concentrations for 1 hour at $4^{\circ} \mathrm{C}$. Then cells were washed three times with cold PBS, incubated with mouse anti-human c-myc antibody (Roche Diagnostics, Mannheim, Germany) for 1 hour at $4^{\circ} \mathrm{C}$, and labelled with secondary goat anti-mouse IgG-RPE antibody (Becton Dickinson, Mountain View, CA, USA) for 1 hour in the darkness at $4^{\circ} \mathrm{C}$. Cells were washed again and resuspended in $200 \mu \mathrm{l}$ PBS containing propidium iodide $(1 \mu \mathrm{g} / \mathrm{ml})$ (Sigma Aldrich, St. Louis, MO, USA) to exclude dead cells. Mean fluorescence intensity (MFI) values were determined by flow cytometry (FACS Calibur) and the binding affinity (Kd), defined as the half-maximal saturation concentration, was calculated using the integrated CellQuest Pro software (BD Biosciences, Heidelberg, Germany).

To test competitive binding, $\mathrm{LNCaP}$ cells were pre-incubated with increasing concentrations of the murine anti-PSMA mAb 3/F11 or J591. Then $5 \mu \mathrm{g} / \mathrm{ml}$ of the humanized/de-immunized scFv hD71(VL-VH) was added to the cells for 1 hour on ice and detected using the secondary antibodies rat anti-human c-myc mAb (Bio-Rad AbD Serotec GmbH, Puchheim, Germany) and goat anti-rat IgG RPE (Thermo Fisher Scientific) for an additional 40 minutes on ice before flow cytometry.

In vitro cytotoxicity. WST-1 cell viability assay (Roche Diagnostics, Indianapolis, IN, USA) was performed to determine the cytotoxic activity of the immunotoxins. $1.5 \times 10^{4}$ cells/well in a 96 -well plate were treated with different concentrations of anti-PSMA immunotoxins alone for 24,48 , and 72 hours. After that, plates were incubated with WST-1 reagent and the optical density was measured at $450 \mathrm{~nm}$ (Ref. $690 \mathrm{~nm}$; ELISA reader, BMG Labtech, Ortenberg, Germany). The $\mathrm{IC}_{50}$ values, defined as a $50 \%$ decrease in cell viability, were estimated utilizing non-linear regression fit with the equation: [log (inhibitor) vs. response (three parameters)] (GraphPad Prism 6 Software; GraphPad Inc., San Diego, CA, USA) as described elsewhere (6).

\section{Results}

Generation, purification and binding of the humanized/deimmunized anti-PSMA scFv. The humanized/de-immunized anti-PSMA scFv hD7-1(VL-VH) was cloned into the vector pHOG21, periplasmatically expressed in E. coli and purified via IMAC. The high-grade purity of the $32 \mathrm{kDa}$ protein was assessed by SDS-PAGE (Figure 2A). The scFv in the VL-VH orientation exhibited a higher binding to PSMA-positive LNCaP and C4-2 cells $\left(\mathrm{K}_{\mathrm{d}}=4.7 \mathrm{nM}\right.$ and $3.7 \mathrm{nM}$, respectively, Figure 2B) compared to the $\mathrm{scFv}$ in the $\mathrm{VH}-\mathrm{VL}$ orientation (data not shown). No binding was detected to PSMA-negative DU145 cells (Figure 2C). Binding inhibition of the scFv hD7$1(\mathrm{VL}-\mathrm{VH})$ with the parental murine anti-PSMA mAb 3/F11 proved that the $\mathrm{ScFv}$ retained binding to the same PSMA epitope. No binding inhibition was seen with the anti-PSMA control mAb J591, which is known to bind to a different PSMA epitope (Figure 2D) (8). Due to its strong and specific PSMA binding, the scFv hD7-1(VL-VH) was identified as a suitable candidate for the construction of the immunotoxins.

Generation, purification and binding of the humanized/deimmunized anti-PSMA immunotoxins. Genes of native toxic domain PE40 and of the de-immunized variants PE24 and PE24mut were inserted C-terminally to the scFv hD7-1(VL$\mathrm{VH})$ in the vector $\mathrm{pHOG} 21$. All immunotoxins were 


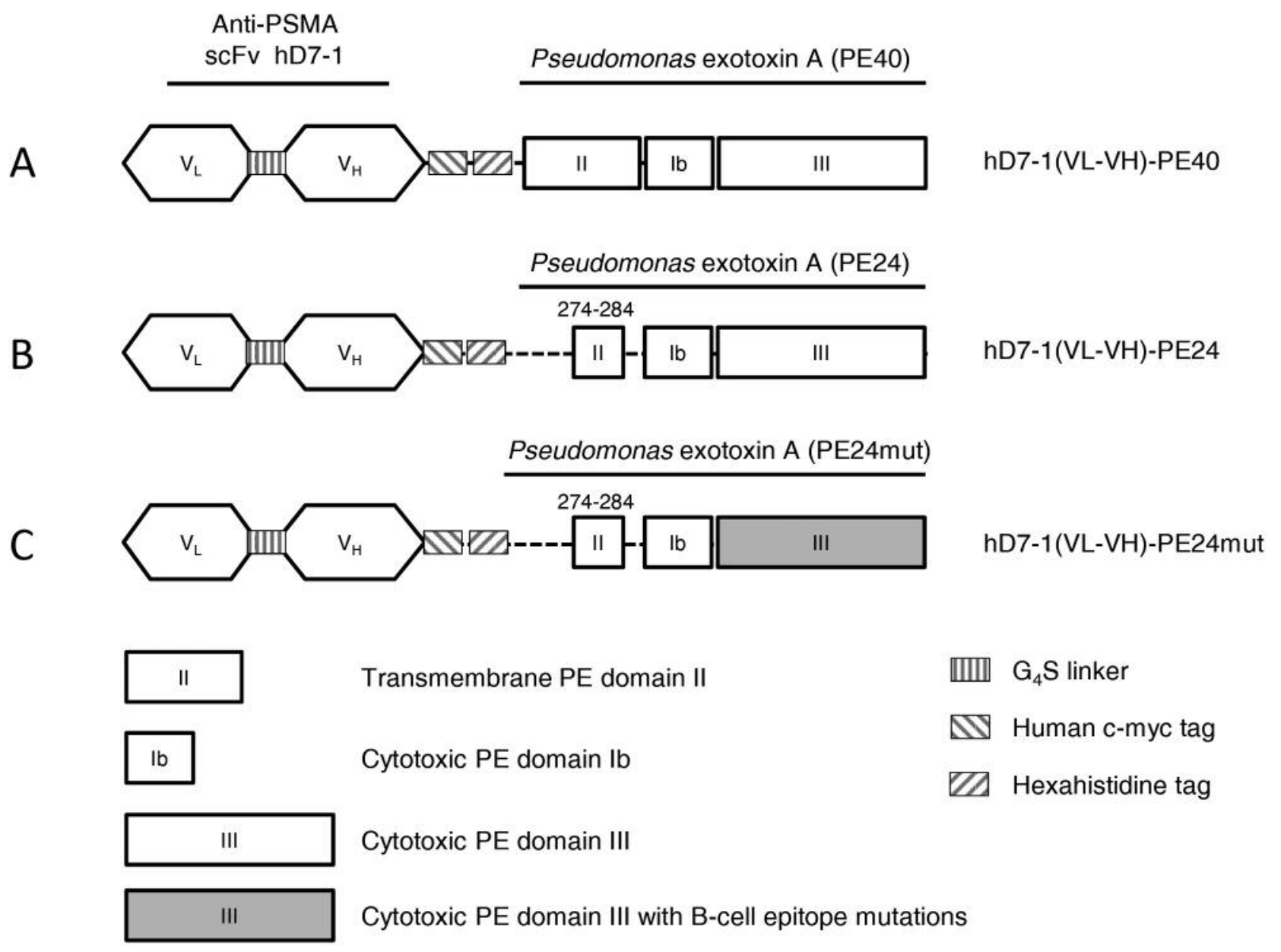

Figure 1. Schematic representation of the de-immunized anti-prostate specific membrane antigen (PSMA) immunotoxin. A: hD7-1(VL-VH)-PE40 containing the original cytotoxic Pseudomonas exotoxin A domain (PE40) consisting of the domains II, Ib and III, B: hD7-1(VL-VH)-PE24 containing the toxic domain PE24 consisting of the deleted PE domain II (with exception of the furin-cleavable linker (amino acids 274-284), Ib and III, and C: hD7-1(VL-VH)-PE24mut with additional mutations in immunodominant B-cell epitopes within domain III (R427A, R458A, D463A, $R 467 A, R 490 A, R 505 A, R 538 A$ ). $G_{4} S$ linker, protein linker (amino acids $G G G G S$ ); scFv, single chain; $V_{L}$, variable domain of the antibody light chain; $V_{H}$ variable domain of the antibody heavy chain.

bacterially expressed and purified in the same way as the scFv. The immunotoxin hD7-1(VL-VH)-PE40 with a molecular weight of about $72 \mathrm{kDa}$ was characterized by SDS-PAGE. The immunotoxins hD7-1(VL-VH)-PE24 and hD7-1(VL-VH)-PE24mut showed an expected molecular size of about $55 \mathrm{kDa}$ (Figure 3A).

The flow cytometric experiments demonstrated that all immunotoxins were capable of specific and efficient binding to PSMA-positive androgen-sensitive $\mathrm{LNCaP}$ and androgenresistant C4-2 cells with comparable $\mathrm{K}_{\mathrm{d}}$ values. With the immunotoxin hD7-1(VL-VH)-PE40, Kd values of $26.4 \mathrm{nM}$ and $9.2 \mathrm{nM}$ for $\mathrm{LNCaP}$ and $\mathrm{C} 4-2$ cells were yielded. With hD7-1(VL-VH)-PE24, Kd values of $10.1 \mathrm{nM}$ and $6.1 \mathrm{nM}$ and with D7-1(VL-VH)-PE24mut $\mathrm{K}_{\mathrm{d}}$ values of $20.9 \mathrm{nM}$ and
10.6 $\mathrm{nM}$ were achieved for $\mathrm{LNCaP}$ and C4-2 cells, respectively (Figure 3B). Flow cytometric analysis of the immunotoxins on PSMA-negative DU145 cells revealed no binding (Figure 3C).

Cytotoxicity of humanized/de-immunized immunotoxins. The immunotoxins hD7-1(VL-VH)-PE40, hD7-(VL-VH)-PE24 and hD7-1(VL-VH)-PE24mut were tested for specific cytotoxic activity against PSMA-positive $\mathrm{LNCaP}$ and C4-2 cells, as well as PSMA-negative DU145 cells. As demonstrated in Figure 4, $\mathrm{IC}_{50}$ values of $55 \mathrm{pM}$ and $25 \mathrm{pM}$ against $\mathrm{LNCaP}$ and of $37 \mathrm{pM}$ and $6 \mathrm{pM}$ against C4-2 cells for $\mathrm{hD} 7-1(\mathrm{VL}-\mathrm{VH})-\mathrm{PE} 40$ were reached after 48- and 72-h incubation, respectively. In addition, high and specific 
A

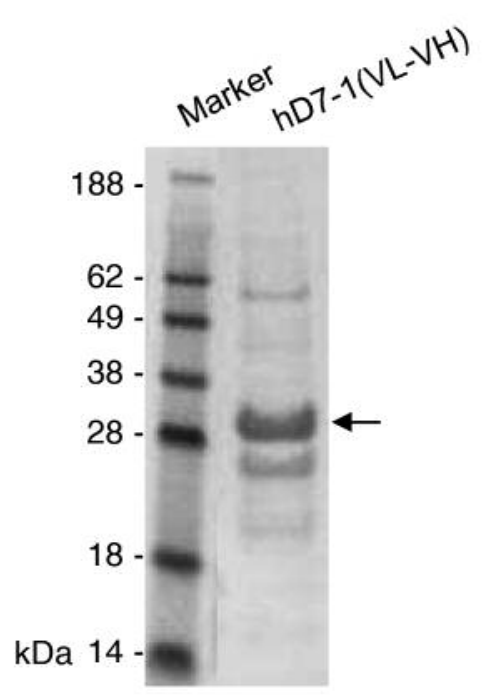

C

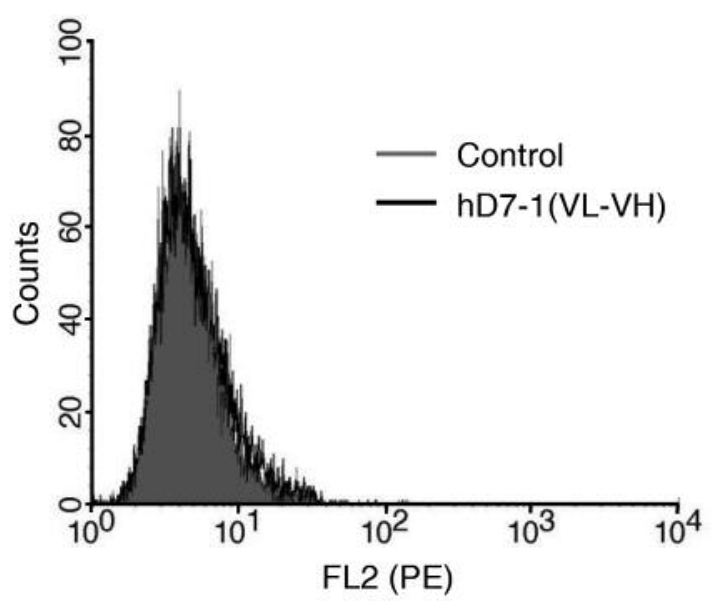

B
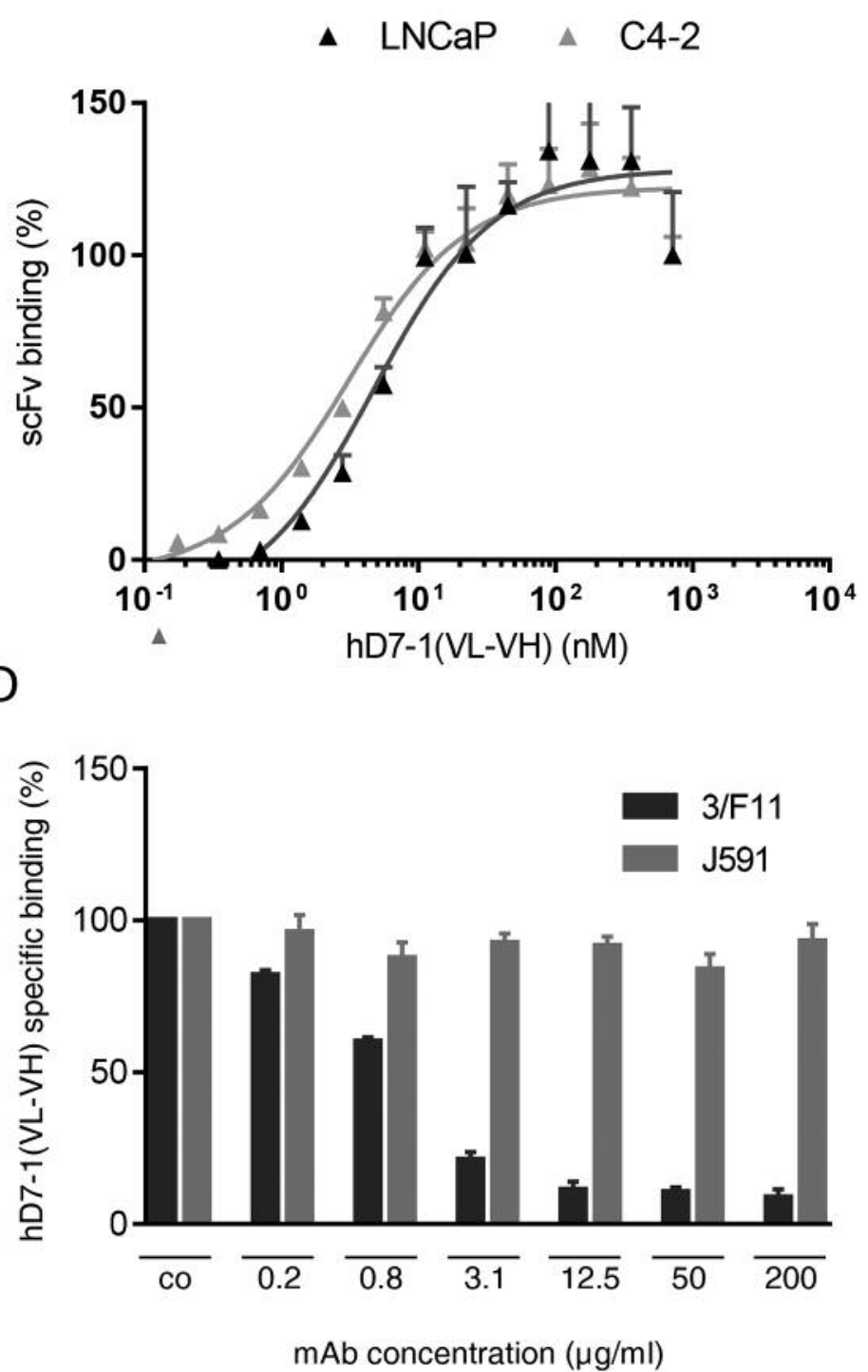

Figure 2. A: Purified humanized/de-immunized single chain antibody fragment (scFv) hD7-1(VL-VH) by sodium dodecyl sulfate-polyacrylamide gel (SDS-PAGE) electrophoresis. B: Binding of hD7-1(VL-VH) to prostate-specific membrane antigen (PSMA)-expressing LNCaP and C4-2 prostate cancer cells by flow cytometry. Data represent three independent experiments $\pm S E M$. C: Binding of $h D 7-1(V L-V H)$ at saturation concentration to PSMA-negative DU145 cells by flow cytometry. D: Binding inhibition of hD7-1(VL-VH) by the parental murine monoclonal antibody (mAb) 3/F11 and the control mAb J591.

cytotoxicity of hD7-1(VL-VH)-PE24 was achieved. With $\mathrm{IC}_{50}$ values of 121 and $82 \mathrm{pM}$ against $\mathrm{LNCaP}$ and of $126 \mathrm{pM}$ and $24 \mathrm{pM}$ against C4-2 cells after 48 and $72 \mathrm{~h}$, respectively, it showed about 2- to 5-fold reduced cytotoxicity compared to the immunotoxin hD7-1(VL-VH)-PE40 containing the complete transmembrane domain II. The immunotoxin hD71(VL-VH)-PE24mut, additionally containing mutated B- and T-cell epitopes within domain III, was shown to be less cytotoxic, with $\mathrm{IC}_{50}$ values of $180 \mathrm{pM}$ and $138 \mathrm{pM}$ against $\mathrm{LNCaP}$ and of 39 and $42 \mathrm{pM}$ against C4-2 cells. No cytotoxicity was seen for any of the tested immunotoxins against DU145 cells (Figure 4C). Taken together, our findings show that de-immunization of the PE toxin domain led to a retention of specificity, however, it also slightly reduced cytotoxicity against PSMA-expressing prostate cancer cells. 
A

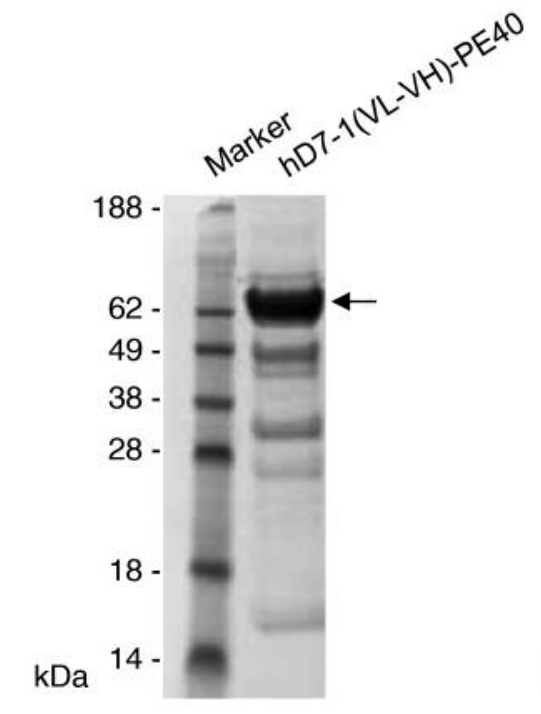

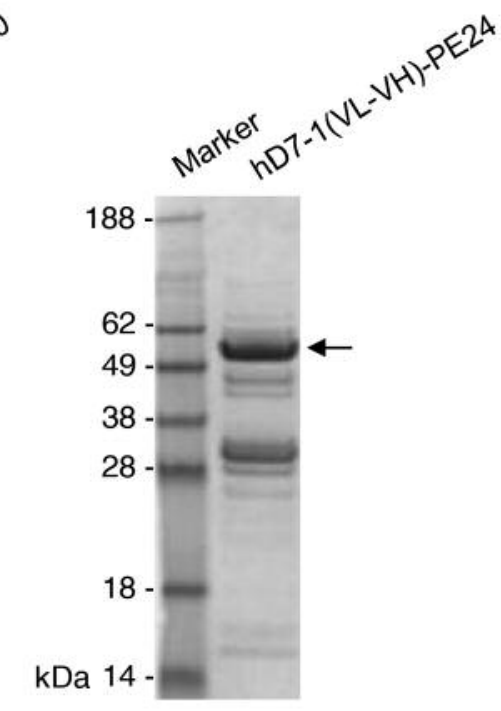

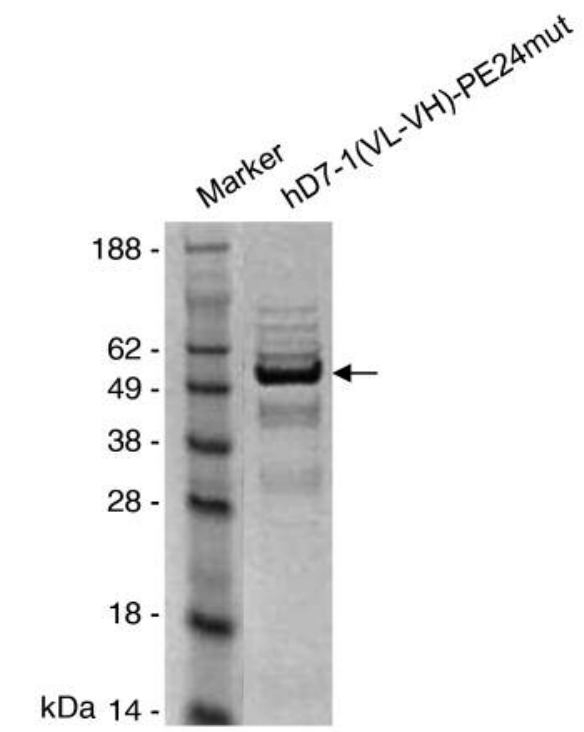

B
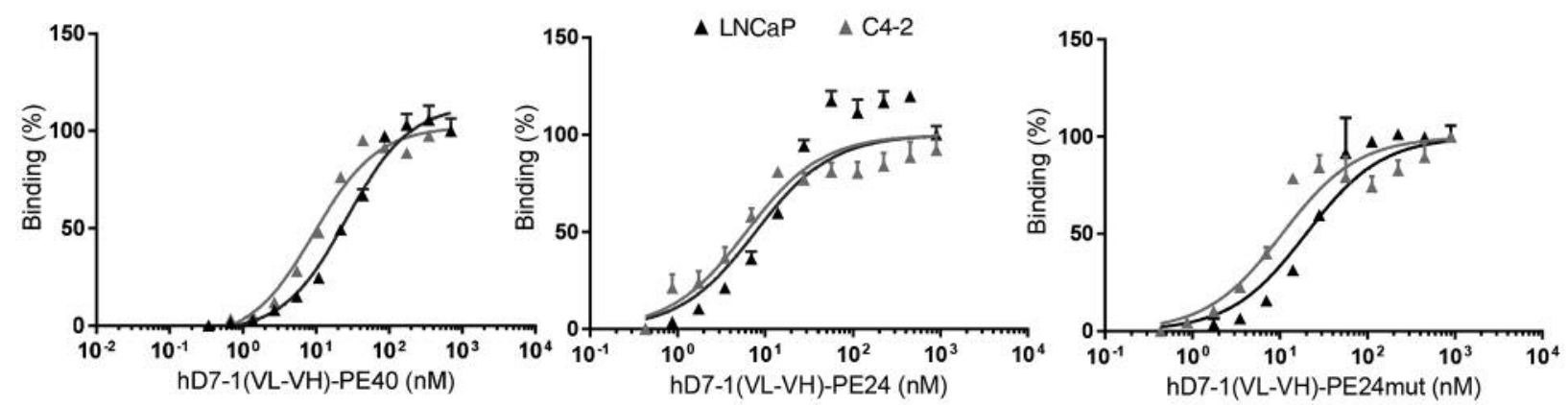

C
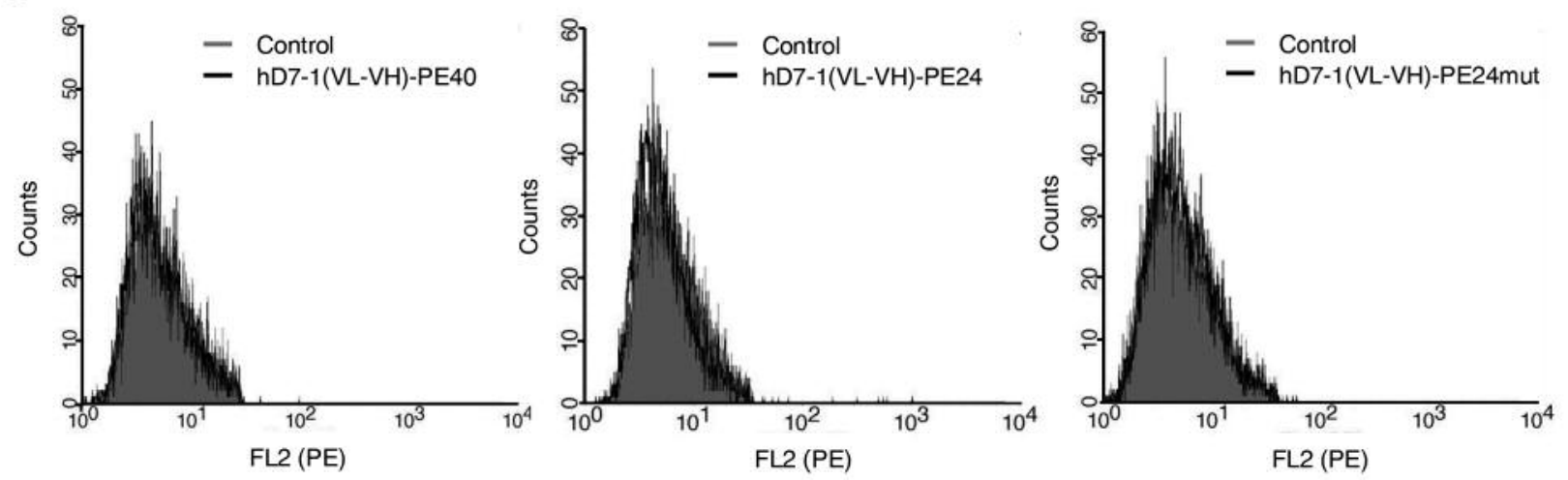

Figure 3. A: High purity of the de-immunized immunotoxins by sodium dodecyl sulfate polyacrylamide gel electrophoresis (SDS-PAGE). B: Flow cytometric analysis of the immunotoxin-specific binding to LNCaP and C4-2 cells by flow cytometry, data represent three independent experiments \pm SEM. C: Binding of the immunotoxins to prostate specific membrane antigen (PSMA)-negative DU145 cells by flow cytometry.

\section{Discussion}

The goal of the present work was the construction of antiPSMA immunotoxins with low immunogenicity that could serve as future therapeutic agents against prostate cancer still remaining incurable in advanced stages. Firstly, we used a humanized/de-immunized variant of our murine $\mathrm{scFv}$ D7 (VL-VH) $(6,23)$ to avoid immunogenicity of the PSMAbinding domain. The humanized/de-immunized scFv hD7$1(\mathrm{VL}-\mathrm{VH})$ showed the same PSMA-epitope binding as its 


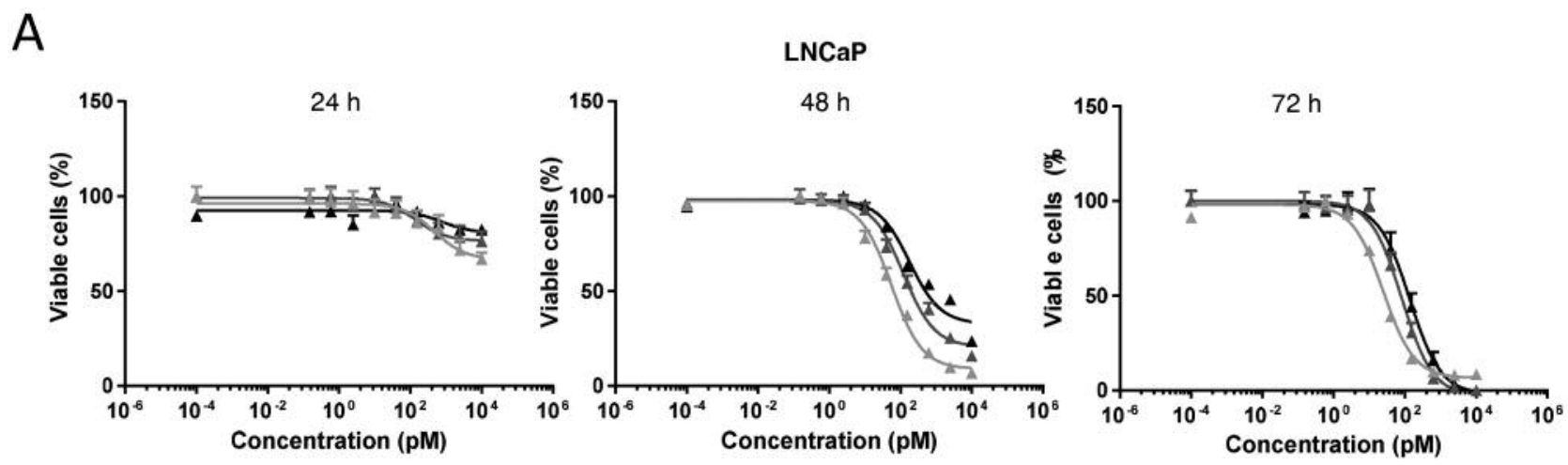

B

C4-2
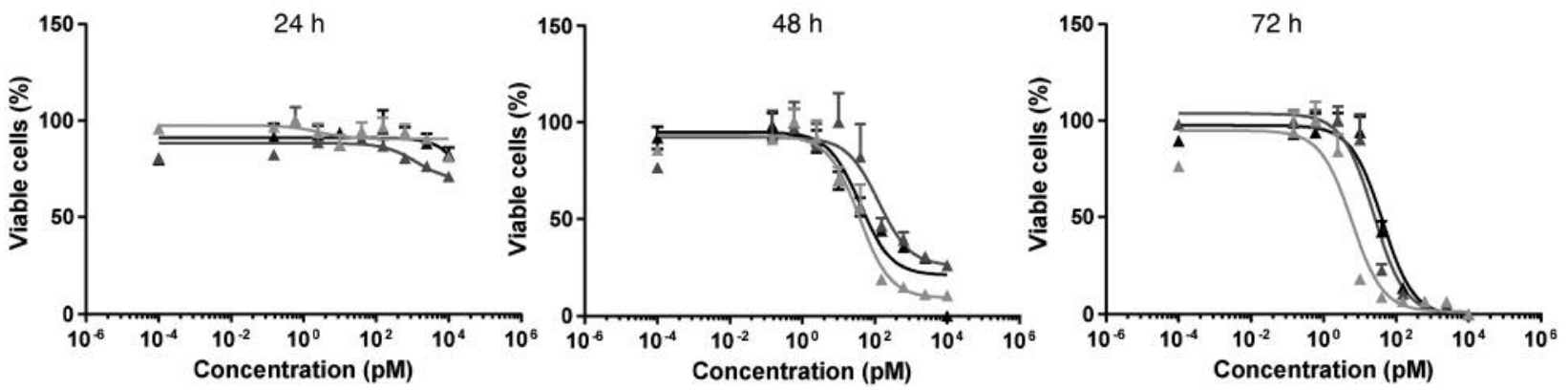

C

DU145
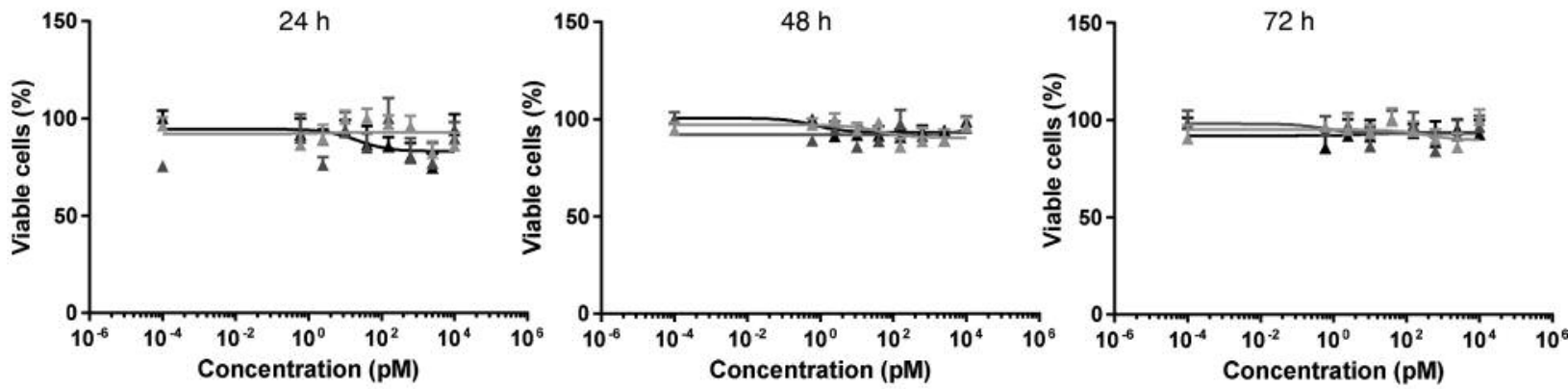

4 hD7-1(VL-VH)-PE40

A hD7-1(VL-VH)-PE24

- hD7-1(VL-VH)-PE24mut

Figure 4. Cytotoxic efficacy of the de-immunized immunotoxins on A: LNCaP, B:C4-2, and C: DU145 cells after 24, 48 and 72 h as measured by WST-1 viability assay.

parental murine scFv D7(VL-VH). Moreover, our data demonstrate that this scFv binds very strongly, with $\mathrm{Kd}$ values in the low nanomolar concentration. Therefore, hD7$1(\mathrm{VL}-\mathrm{VH})$ was proven to be particularly suitable for the development of targeted immunotoxins as it was shown that
$\mathrm{scFv}$ with binding affinities in the nanomolar range have optimal tumor accumulation and penetration (24-26).

PE-based immunotoxins with truncated $40 \mathrm{kDa}$ or $38 \mathrm{kDa}$ domains have been previously used in preclinical and clinical trials against various tumors (27-29). There are currently 
ongoing phase I and II clinical studies with PE38-based immunotoxins against hematological and solid tumors. Wayne et al. characterized the safety profile of CD22-PE38 (HA22, moxetumomab pasudotox) against acute lymphoblastic leukemia in 55 patients and demonstrated moderate immunogenicity. Continuous administration (10 doses of 50 $\mu \mathrm{g} / \mathrm{kg}$ ) of this immunotoxin, however, resulted in hemolytic uremic syndrome and thrombotic microangiopathy in six out of 14 patients (30). Kreitman et al. also showed that moxetumomab pasudotox was immunogenic in 10 out of 28 examined patients developing ADA during the study (31). The application of immunotoxins against solid tumors is much more complex than against hematological malignancies. ADA formation is present as early as after one therapy cycle due to the strong patient's immune system, which does not occur or is very rare in patients suffering from hematological tumors (32). In a phase I study, Hassan et al. demonstrated that the majority of patients $(n=34)$ produced ADA with grade 3 toxicities (related to fatigue, back pain, hypoalbuminemia, hypotension) even after a single dose of SS1P immunotoxin (SS1-dsFv-PE38) against mesothelinpositive solid tumors $(33,34)$.

In order to diminish immunogenicity of the PE domains, structural analyses have been undertaken to identify and mutate immunodominant epotipes. Mazor et al. described that deletion of domain II can silence the immunodominant B- and T-cell epitopes, and thereby reduce the immunogenicity of the toxin domain and diminish the production of ADA $(11,35)$. Furthermore, it has been shown that structural and functional changes within the translocation domain II of PE result in enhanced stability (36, 37). Weldon et al. constructed an anti-CD22 immunotoxin (HA22-LR) against hematological malignancies (37) and an anti-mesothelin immunotoxin (SS1-LR-GSS) against solid tumors (38) with lysosome protease resistance. They demonstrated that deletion of domain II or insertion of specific mutations within domain III led to enhanced stability without loss of its activity (3638). Moreover, they found that deletion of domain II, with exception of the furin-cleavable site (aa 274-284), resulted not only in higher stability but also in an approximately twofold higher cytotoxicity compared to the initial construct (37). According to these publications, we generated the humanized/de-immunized anti-PSMA immunotoxin hD71(VL-VH)-PE24 with the deleted transmembrane domain II retaining the furin-cleavable site. The specific PSMA binding was maintained and high and specific cytotoxicity against PSMA-expressing prostate cancer cells was demonstrated. However, the partial deleted transmembrane domain II within the hD7-1(VL-VH)-PE24 immunotoxin resulted in reduced cytotoxicity, indicating that these changes might contribute to a diminished release of the toxin domain into the cytosol. Further experiments will be conducted to analyze the cellular fate of the immunotoxin in detail. The reduced cytotoxicity is in contrast to the publication of Weldon et al., where an approximately twofold enhanced cytotoxicity of the deimmunized immunotoxin HA22-LR compared to the immunotoxin with the PE38 domain was described (37).

Besides deleting $\mathrm{B}$ - and T-cell epitopes within the transmembrane domain II, it was shown that specific mutations within the PE domain III silenced additional Bcell epitopes and led to reduced immunogenicity $(20,21)$. Liu et al. found that mutations of seven immunodominant Bcell epitopes to alanine (R457A, R458A, D463A, R467A, R490A, R505A, R538A) led to partial or complete disappearance of binding of neutralizing antibodies $(20,39)$. We implemented these mutations in our immunotoxin hD71(VL-VH)-PE24mut and found that these mutations did not affect binding to prostate cancer cells. We also obtained specific cytotoxicity comparable with that of the immunotoxin hD7-1(VL-VH)-PE40 ( $\mathrm{IC}_{50}$ values in picomolar range). Further work is needed to examine the antitumor activity in in vivo models.

In our present work, we generated highly specific and cytotoxic de-immunized anti-PSMA immunotoxins that might be suitable for a future improved targeted-therapy of advanced prostate cancer.

\section{Acknowledgements}

This work was supported by the Deutsche Krebshilfe (Grant No. 110993 to Philipp Wolf). We thank HD Pharma (Ladenburg, Germany) for providing the humanized sequence of the anti-PSMA scFv.

\section{References}

1 Flores O, Santra S, Kaittanis C, Bassiouni R, Khaled AS, Khaled AR, Grimm J and Perez JM: PSMA-targeted theranostic nanocarrier for prostate cancer. Theranostics 7(9): 2477-2494, 2017.

2 Eder M, Eisenhut M, Babich $\mathrm{J}$ and Haberkorn U: PSMA as a target for radiolabelled small molecules. Eur J Nucl Med Mol Imaging 40(6): 819-823, 2013.

3 Hrkach J, Von Hoff D, Mukkaram Ali M, Andrianova E, Auer J, Campbell T, De Witt D, Figa M, Figueiredo M, Horhota A, Low S, McDonnell K, Peeke E, Retnarajan B, Sabnis A, Schnipper E, Song JJ, Song YH, Summa J, Tompsett D, Troiano G, Van Geen Hoven T, Wright J, LoRusso P, Kantoff PW, Bander NH, Sweeney C, Farokhzad OC, Langer R and Zale S: Preclinical development and clinical translation of a PSMA-targeted docetaxel nanoparticle with a differentiated pharmacological profile. Sci Transl Med 4(128): 128ra139, 2012.

4 Bouchelouche K, Choyke PL and Capala J: Prostate-specific membrane antigen- a target for imaging and therapy with radionuclides. Discov Med 9(44): 55-61, 2010

5 Tagawa ST, Beltran H, Vallabhajosula S, Goldsmith SJ, Osborne J, Matulich D, Petrillo K, Parmar S, Nanus DM and Bander NH: Anti-prostate-specific membrane antigen-based radioimmunotherapy for prostate cancer. Cancer 116(4 Suppl): 1075-1083, 2010. 
6 Michalska M, Schultze-Seemann S, Bogatyreva L, Hauschke D Wetterauer $\mathrm{U}$ and Wolf $\mathrm{P}$ : In vitro and in vivo effects of a recombinant anti-PSMA immunotoxin in combination with docetaxel against prostate cancer. Oncotarget 7(16): 2253122542, 2016.

7 Wolf P, Gierschner D, Buhler P, Wetterauer U and Elsasser-Beile $\mathrm{U}$ : A recombinant PSMA-specific single-chain immunotoxin has potent and selective toxicity against prostate cancer cells. Cancer Immunol Immunother 55(11): 1367-1373, 2006.

8 Wolf P, Freudenberg N, Buhler P, Alt K, Schultze-Seemann W, Wetterauer $U$ and Elsasser-Beile U: Three conformational antibodies specific for different PSMA epitopes are promising diagnostic and therapeutic tools for prostate cancer. Prostate 70(5): 562-569, 2010.

9 Elsasser-Beile U, Wolf P, Gierschner D, Buhler P, SchultzeSeemann W and Wetterauer U: A new generation of monoclonal and recombinant antibodies against cell-adherent prostatespecific membrane antigen for diagnostic and therapeutic targeting of prostate cancer. Prostate 66(13): 1359-1370, 2006.

10 Michalska M and Wolf P: Pseudomonas exotoxin A: Optimized by evolution for effective killing. Front Microbiol 6: 963, 2015

11 Mazor R, Onda M and Pastan I: Immunogenicity of therapeutic recombinant immunotoxins. Immunol Rev 270(1): 152-164, 2016.

12 Schellekens H: How to predict and prevent the immunogenicity of therapeutic proteins. Biotechnol Annu Rev 14: 191-202, 2008.

13 Malucchi S, Sala A, Gilli F, Bottero R, Di Sapio A, Capobianco $\mathrm{M}$ and Bertolotto A: Neutralizing antibodies reduce the efficacy of betain during treatment of multiple sclerosis. Neurology 62(11): 2031-2037, 2004.

14 Bera TK, Onda M, Kreitman RJ and Pastan I: An improved recombinant $\mathrm{FAB}$-immunotoxin targeting $\mathrm{CD} 22$-expressing malignancies. Leuk Res 38(10): 1224-1229, 2014.

15 Holliger P and Hudson PJ: Engineered antibody fragments and the rise of single domains. Nat Biotechnol 23(9): 1126-1136, 2005.

16 Shan L, Liu Y and Wang P: Recombinant immunotoxin therapy of solid tumors: Challenges and strategies. J Basic Clin Med 2(2): 1-6, 2013.

17 Tsutsumi Y, Onda M, Nagata S, Lee B, Kreitman RJ and Pastan I: Site-specific chemical modification with polyethylene glycol of recombinant immunotoxin anti-TAC(Fv)-PE38 (LMB-2) improves antitumor activity and reduces animal toxicity and immunogenicity. Proc Natl Acad Sci USA 97(15): 8548-8553, 2000.

18 Filpula D, Yang K, Basu A, Hassan R, Xiang L, Zhang Z, Wang M, Wang QC, Ho M, Beers R, Zhao H, Peng P, Zhou J, Li X, Petti G, Janjua A, Liu J, Wu D, Yu D, Zhang Z, Longley C, FitzGerald D, Kreitman RJ and Pastan I: Releasable pegylation of mesothelin targeted immunotoxin SS1P achieves single dosage complete regression of a human carcinoma in mice. Bioconjug Chem 18(3): 773-784, 2007.

19 Benhar I, Wang QC, FitzGerald D and Pastan I: Pseudomonas exotoxin A mutants. Replacement of surface-exposed residues in domain III with cysteine residues that can be modified with polyethylene glycol in a site-specific manner. J Biol Chem 269(18): 13398-13404, 1994.

20 Liu W, Onda M, Lee B, Kreitman RJ, Hassan R, Xiang L and Pastan I: Recombinant immunotoxin engineered for low immunogenicity and antigenicity by identifying and silencing human B-cell epitopes. Proc Natl Acad Sci USA 109(29): 1178211787, 2012.
21 Onda M, Nagata S, FitzGerald DJ, Beers R, Fisher RJ, Vincent JJ, Lee B, Nakamura M, Hwang J, Kreitman RJ, Hassan R and Pastan I: Characterization of the B-cell epitopes associated with a truncated form of Pseudomonas exotoxin (PE38) used to make immunotoxins for the treatment of cancer patients. J Immunol 177(12): 8822-8834, 2006.

22 Yeung VP, Chang J, Miller J, Barnett C, Stickler M and Harding FA: Elimination of an immunodominant $\mathrm{CD}^{+}{ }^{+} \mathrm{T}$-cell epitope in human IFN-beta does not result in an in vivo response directed at the subdominant epitope. J Immunol 172(11): 6658-6665, 2004.

23 Wolf $\mathrm{P}$, Alt K, Wetterauer D, Buhler P, Gierschner D, Katzenwadel A, Wetterauer U and Elsasser-Beile U: Preclinical evaluation of a recombinant anti-prostate-specific membrane antigen single-chain immunotoxin against prostate cancer. $\mathbf{J}$ Immunother 33(3): 262-271, 2010.

24 Adams GP, Schier R, Marshall K, Wolf EJ, McCall AM, Marks JD and Weiner LM: Increased affinity leads to improved selective tumor delivery of single-chain Fv antibodies. Cancer Res 58(3): 485-490, 1998.

25 Adams GP, Schier R, McCall AM, Simmons HH, Horak EM, Alpaugh RK, Marks JD and Weiner LM: High affinity restricts the localization and tumor penetration of single-chain Fv antibody molecules. Cancer Res 61(12): 4750-4755, 2001.

26 Suksanpaisan L, Russell SJ and Peng KW: High scFv-receptor affinity does not enhance the antitumor activity of HER2retargeted measles virus. Cancer Gene Ther 21(6): 256-260, 2014.

27 Weldon JE and Pastan I: A guide to taming a toxin recombinant immunotoxins constructed from Pseudomonas exotoxin A for the treatment of cancer. FEBS J 278(23): 46834700, 2011.

28 Risberg K, Fodstad O and Andersson Y: Immunotoxins: A promising treatment modality for metastatic melanoma? Ochsner J 10(3): 193-199, 2010.

29 Kreitman RJ: Immunotoxins for targeted cancer therapy. AAPS J 8(3): E532-551, 2006.

30 Wayne AS, Shah NN, Bhojwani D, Silverman LB, Whitlock JA, Stetler-Stevenson M, Sun W, Liang M, Yang J, Kreitman RJ, Lanasa MC and Pastan I: Phase I study of the anti-CD22 immunotoxin moxetumomab pasudotox for childhood acute lymphoblastic leukemia. Blood 130(14): 1620-1627, 2017.

31 Kreitman RJ, Tallman MS, Robak T, Coutre S, Wilson WH, Stetler-Stevenson M, Fitzgerald DJ, Lechleider R and Pastan I: Phase I trial of anti-CD22 recombinant immunotoxin moxetumomab pasudotox (CAT-8015 or HA22) in patients with hairy cell leukemia. J Clin Oncol 30(15): 1822-1828, 2012.

32 Li M, Liu ZS, Liu XL, Hui Q, Lu SY, Qu LL, Li YS, Zhou Y, Ren $\mathrm{HL}$ and $\mathrm{Hu} \mathrm{P}$ : Clinical targeting recombinant immunotoxins for cancer therapy. Onco Targets Ther 10: 3645-3665, 2017.

33 Hassan R, Sharon E, Thomas A, Zhang J, Ling A, Miettinen M, Kreitman RJ, Steinberg SM, Hollevoet K and Pastan I: Phase 1 study of the antimesothelin immunotoxin SS1P in combination with pemetrexed and cisplatin for front-line therapy of pleural mesothelioma and correlation of tumor response with serum mesothelin, megakaryocyte potentiating factor, and cancer antigen 125. Cancer 120(21): 3311-3319, 2014.

34 Hassan R, Bullock S, Premkumar A, Kreitman RJ, Kindler H, Willingham MC and Pastan I: Phase I study of SS1P, a recombinant anti-mesothelin immunotoxin given as a bolus i.v. 
Infusion to patients with mesothelin-expressing mesothelioma, ovarian, and pancreatic cancers. Clin Cancer Res 13(17): 51445149, 2007.

35 Mazor R, Eberle JA, Hu X, Vassall AN, Onda M, Beers R, Lee EC, Kreitman RJ, Lee B, Baker D, King C, Hassan R, Benhar I and Pastan I: Recombinant immunotoxin for cancer treatment with low immunogenicity by identification and silencing of human T-cell epitopes. Proc Natl Acad Sci USA 111(23): 85718576, 2014.

36 Hansen JK, Weldon JE, Xiang L, Beers R, Onda M and Pastan $\mathrm{I}$ : A recombinant immunotoxin targeting CD22 with low immunogenicity, low nonspecific toxicity, and high antitumor activity in mice. J Immunother 33(3): 297-304, 2010.

37 Weldon JE, Xiang L, Chertov O, Margulies I, Kreitman RJ, FitzGerald DJ and Pastan I: A protease-resistant immunotoxin against CD22 with greatly increased activity against CLL and diminished animal toxicity. Blood 113(16): 3792-3800, 2009.
38 Weldon JE, Xiang L, Zhang J, Beers R, Walker DA, Onda M, Hassan R and Pastan I: A recombinant immunotoxin against the tumor-associated antigen mesothelin reengineered for high activity, low off-target toxicity, and reduced antigenicity. Mol Cancer Ther 12(1): 48-57, 2013.

39 King C, Garza EN, Mazor R, Linehan JL, Pastan I, Pepper M and Baker D: Removing T-cell epitopes with computational protein design. Proc Natl Acad Sci USA 111(23): 8577-8582, 2014.

Received October 11, 2017

Revised October 27, 2017

Accepted November 1, 2017 